\title{
Erratum: One-dimensional mimicking of electronic structure: The case for exponentials [Phys. Rev. B 91, 235141 (2015)]
}

Thomas E. Baker, E. Miles Stoudenmire, Lucas O. Wagner, Kieron Burke, and Steven R. White

(Received 5 March 2016; published 31 March 2016)

DOI: 10.1103/PhysRevB.93.119912

After publication of our paper, we noticed three small typos. None of the conclusions of the paper are affected, and all benchmark numbers are correct.

The typos are listed as follows:

(i) In Table I: the parameter $\sigma$ for $e_{\mathrm{C}}^{\mathrm{pol}}$ lacks a negative sign (it reads 4.32708 and should be -4.32708 ). The $\beta$ parameter for the unpolarized fit in Table I also lacks a negative sign (it reads 1.00077 but should be -1.00077 ). A corrected table is provided below.

\begin{tabular}{lcccccc}
\hline \hline & $\alpha$ & $\beta$ & $\gamma$ & $\delta$ & $\eta$ & $\sigma$ \\
\hline$e_{c}^{\text {unpol }}$ & 2 & -1.00077 & 6.26099 & -11.9041 & 9.62614 & -1.48334 \\
$e_{c}^{\text {pol }}$ & 180.891 & -541.124 & 651.615 & -356.504 & 88.0733 & -4.32708 \\
\hline \hline
\end{tabular}

(ii) The figure caption for Fig. 2 of the original paper should be the energy per particle, $e / n$. An updated version appears in Fig. 1 here with a corrected caption. The $y$ axis is also changed to the per particle label.

(iii) The figure caption for Fig. 4 of the original paper should be the energy per particle, $e / n$. An updated version appears in Fig. 2 here with a corrected caption. The $y$ axis is also changed to the per particle label.

We wish to amend these points here, and we apologize for missing these small but noticeable typos.

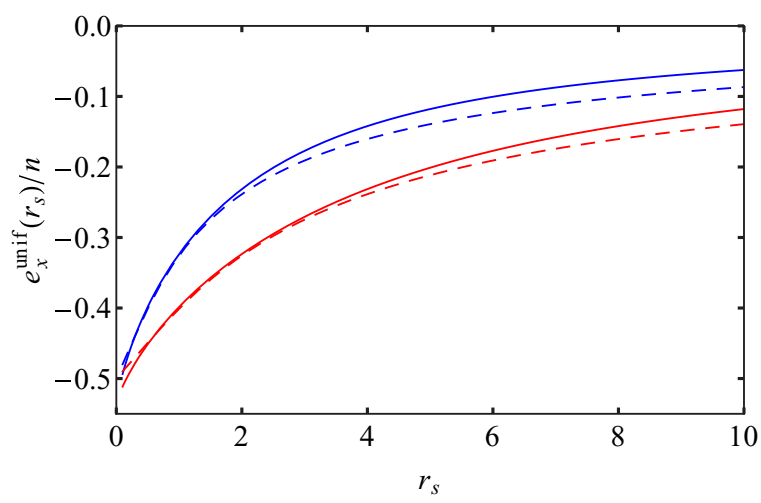

FIG. 1. Exchange energy densities per particle of the uniform gas for the exponential (solid lines), both unpolarized (blue) and fully polarized (red). Dashed lines show soft-Coulomb results.

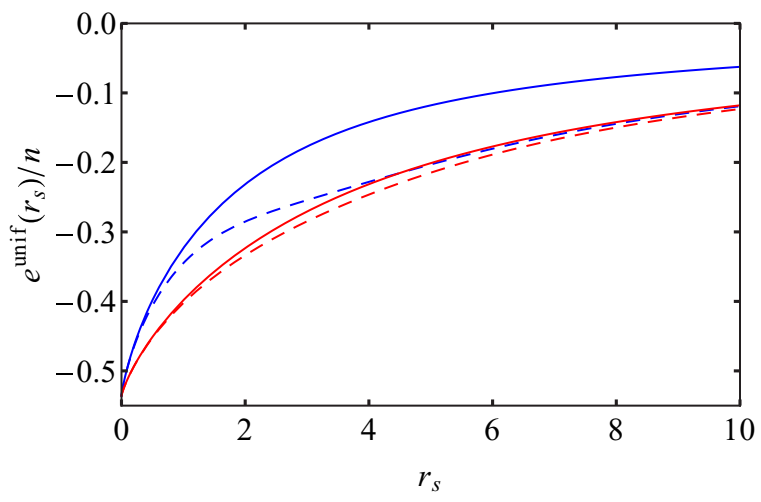

FIG. 2. XC energy per particle, $e_{\mathrm{xC}} / n$ (dashed), for the unpolarized (blue) and fully polarized (red). Solid lines are $e_{\mathrm{X}} / n$ only. 